\title{
Systemic Lupus Erythematosus Associated with Rowell's Syndrome
}

\author{
A Clinical Pathology Conference Held by the Division of Rheumatology at Hospital for Special \\ Surgery
}

\author{
Susan S. Kim, MD • Cynthia Magro, MD • Richard D. Granstein, MD • Anne Bass, MD • Doruk Erkan, MD
}

Received: 8 March 2013/Accepted: 26 July 2013/ Published online: 29 August 2013

(C) Hospital for Special Surgery 2013

Keywords Systemic lupus erythematosus .

Rowell's syndrome

\section{Clinical Presentation}

A 22-year-old man with systemic lupus erythematosus (SLE) presented with 2 weeks of a generalized rash and oral ulcers.

The patient was diagnosed with SLE at the age of 3 based on malar rash, photosensitivity, oral ulcers, and arthritis, which were controlled with chronic low-dose corticosteroids and hydroxychloroquine. At the age of 18 , the patient developed aseptic meningitis and acute left anterior uveitis; both were responsive to increased doses of corticosteroids. He did not follow up with a rheumatologist for approximately 4 years, during which time he took no medication until 4 months prior to admission when he experienced a lupus flare with malar

$\overline{\text { Electronic supplementary material The online version of this article }}$ (doi:10.1007/s11420-013-9356-6) contains supplementary material, which is available to authorized users.

S. S. Kim, MD $(\bowtie) \cdot$ A. Bass, MD $\cdot$ D. Erkan, MD

Department of Rheumatology,

Hospital for Special Surgery,

535 East 70th Street,

New York, NY 10021, USA

e-mail: imsusaki@gmail.com

C. Magro, MD

Department of Pathology and Laboratory Medicine, Weill-Cornell New York-Presbyterian Hospital,

525 East 68th Street,

New York, NY 10065, USA

R. D. Granstein, MD

Department of Dermatology,

Weill Cornell Medical College,

520 East 70th Street,

New York, NY 10065, USA rash, oral ulcers, fatigue, and neuropsychiatric symptoms including severe headache, distal paresthesias, slurred speech, and a subacute decline in mental status (difficulty concentrating, slowing thoughts, word finding difficulties, and memory loss). Laboratory testing at that time revealed a moderately high titer positive antinuclear antibody (ANA), in a coarse speckled pattern, and anti-double-stranded deoxyribonucleic acid (anti-dsDNA) antibody, normal levels of $\mathrm{C} 3$ and $\mathrm{C} 4$ and positive Ro (SS-A), Smith (Sm), U1-ribonucleoprotein, anticardiolipin immunoglobulin A (IgA) (42U), and Beta-2 glycoprotein-I IgA (57U) antibodies. The patient was treated with high-dose corticosteroids, started on hydroxychloroquine $200 \mathrm{mg}$ twice daily, aspirin $81 \mathrm{mg}$, and esomeprazole $40 \mathrm{mg}$ once daily, with clinical improvement. Patient was subsequently tapered down on prednisone therapy accordingly from 60 to $40 \mathrm{mg}$ once daily regimen.

Two weeks prior to admission at our institution, the patient developed new oral ulcers and generalized rash. The rash, which was minimally pruritic, initially appeared on his hands and arms, and then extended to his trunk and face, sparing his lower extremities and buttocks. Small painful vesicular lesions with serous discharge developed on his palms and fingers. His oral ulcers, initially discrete and small, became diffuse and painful and made it difficult for him to eat, as per patient's selfreport. He sought care at an emergency department at another institution where he was treated with intravenous valacycloir for possible disseminated herpes-simplex viral (HSV) infection and with vancomycin for a possible bacterial skin superinfection. Less than $24 \mathrm{~h}$ there, the patient left against medical advice, and presented to our hospital the following day.

The patient denied joint pain, neurological symptoms, fevers or chills, chest pain, shortness of breath, cough, diarrhea, constipation, dysuria, or hematuria. The patient had known allergies to penicillin and erythromycin, but no history of recent exposure to these drugs. His medications were prednisone $40 \mathrm{mg}$ alternating with $20 \mathrm{mg}$ daily, hydroxychloroquine $200 \mathrm{mg}$ twice daily, aspirin $81 \mathrm{mg}$ daily, and esomeprazole 
40mg daily. Family history was significant for a younger sister with SLE. The patient lived with his family and recently finished high school. He denied any alcohol, tobacco, illicit drug use, or any recent sexual contact.

On physical examination, the patient had a temperature of $38.5^{\circ} \mathrm{C}$, blood pressure $118 / 73 \mathrm{mmHg}$, heart rate 84 beats per minute, respiration rate 16 breaths per minute, and oxygen saturation $96 \%$ on room air. He was ill-appearing. Ocular examination was significant for bilateral conjunctival injection. There were erythematous pustules around his eyelid margins with purulent discharge. There were yellow plaques and ulcerations on both his buccal mucosa and hard palate. On his face, he had well-demarcated erythematous plaques with overlying crust in a malar distribution sparing the nasolabial folds. The rash extended over his cheeks, eyebrows, and pinnae of his ears (ESM Picture 1a). There were also erythematous papules and papulopustular lesions on his chest, abdomen, back, and arms. His palms had erythematous macules and papules, some targetoid in shape, with three zones of color as follows: dusky center, skin colored middle rim, and an erythematous periphery (ESM Picture $1 \mathrm{~b}$ ). He had tender, reddish nodules with acral ulcerations. There were well-demarcated areas of erythema on the finger tips with serous subungual discharge. Examination of patient's lower extremities, buttocks, groin, and genitals were normal. There was no synovitis or lymphadenopathy. Cardiopulmonary, abdominal, and neurologic examinations were normal. There was no synovitis, and neurological exam was normal. The initial clinical impression was concern for a disseminated mucocutaneous viral infection from a herpes simplex or varicella zoster virus (VZV), with a bacterial superinfection in this immunosuppressed SLE patient. Other considerations included a possible drug-induced eruption, as well as the possibility of a concomitant SLE process, given the appearance of a classic malar rash and acral ulcerations consistent with chilblains, which were concerning for an underlying vasculitic process.

Initial laboratory values revealed normal white blood cell count, erythrocyte sedimentation rate, C-reactive protein, and renal and liver function tests (Table 1). Tests for human

Table 1 Initial laboratory tests

\begin{tabular}{lll}
\hline & Result & Normal values \\
\hline White blood cell & 5.5 & $3.4-11.2 \times 10^{3} / \mu \mathrm{L}$ \\
$\quad$ Neutrophil & 67.8 & $45.0-75.0 \%$ \\
Lymphocyte & 17.4 & $20.0-50.0 \%$ \\
Monocyte & 14.2 & $2.0-11.0 \%$ \\
Eosinophil & 0.2 & $0.0-5.0 \%$ \\
Basophil & 0.4 & $0.0-1.0 \%$ \\
Hemoglobin & 14.5 & $13.3-17.7 \mathrm{~g} / \mathrm{dl}$ \\
Hematocrit & 41.6 & $40.0-52.0 \%$ \\
Platelet & 239 & $150-450 \times 10^{3} / \mu \mathrm{L}$ \\
Creatinine & 0.60 & $0.64-1.27 \mathrm{mg} / \mathrm{dL}$ \\
Erythrocyte & 30 & $0-15 \mathrm{~mm} / \mathrm{h}$ \\
sedimentation rate & & $0-1 \mathrm{mg} / \mathrm{dl}$ \\
C-reactive protein & 0.7 & $\mathrm{Mg} / \mathrm{dl}$ \\
Spot Prot/Cr & 0.09 & \\
Urinalysis & Trace protein, & \\
& neg RBC, 0WBC, & \\
& neg bacteria & \\
\hline
\end{tabular}

immunodeficiency virus enzyme-linked immunosorbent assay, urine testing for Neisseria gonorrhoeae (GC) and Chlamydia trachomatis, and syphilis screening test (rapid plasma reagin) were negative. Blood and urine cultures drawn at admission and during febrile episodes were negative. Urinalysis, spot protein to creatinine ratio, complement levels, and double-stranded DNA were normal. Other lupus serologies are shown in Table 2.

Intravenous acyclovir $(10 \mathrm{mg} / \mathrm{kg})$ and vancomycin were resumed. Prednisone dose was decreased to $10 \mathrm{mg}$ twice daily. The patient was evaluated by dermatology. Skin scrapings and biopsy were taken for direct fluorescent antibody (DFA) testing, histopathologic analysis, and viral culture for VZV and HSV.

\section{Pathological Discussion}

Under microscopy, the skin showed features most compatible with lupus features of interface dermatitis with epithelial destruction and resultant epidermal attenuation, accompanied by hyperkeratosis and mesenchymal mucin deposition (Fig. 1). Against this classic background of a lupus-like diathesis, there were areas of rather striking suprabasilar lymphocytic satellitosis in direct apposition to necrotic keratinocytes (Fig. 2). These foci of cell-rich interface dermatitis involved sites of antigenic processing, namely the acrosyringium and hair follicle [3]. The area of cytotoxic interface dermatitis with lymphocyte satellitosis to necrotic keratinocytes reflect a Gell and Coombs type IV immune reaction, pathogenically analogous to erythema multiforme (EM) [12]. This cell-mediated response in classical EM is elicited by $\mathrm{T}$ cells responding to a limited triggering antigenic epitope, which include infections (herpes simplex, Mycoplasma pneumoniae) or drugs (antibiotics, antiepileptics, non-steroid antiinflammatory, and sulfonamides). Keratinocytes become antigenic through drug-hapten binding to keratinocytes or in the setting of viral infections molecular mimicry or possible incorporation of viral DNA into the cell rendering it antigenic $[2,8]$.

In this case, the biopsy lacked any cytopathic changes to suggest herpes viral infection to support a concomitant infectious process. The DFA for HSV/VZV were negative. Instead, the biopsy was defined by features consistent with lupus erythematosus (LE), such as mucin deposition in the dermis and follicular plugging with epidermal attenuation, but with a variant histomorphology by virtue of the supervening type IV cytotoxic immune reaction analogous to erythema multiforme-like interface changes. The combination has been referred to as Rowell's syndrome (RS) [9].

Table 2 Rheumatologic serological tests

\begin{tabular}{lll}
\hline Antinuclear antibodies & $1+$ & Negative \\
Anti-double-stranded DNA & Negative & Negative \\
Anti-Ro/La/Sm/RNP & $>100 /<16 />100 />100$ & $<16$ E Units/ml \\
Complement 3 & 121 & $79-152 \mathrm{mg} / \mathrm{dL}$ \\
Complement 4 & 31 & $16-38 \mathrm{mg} / \mathrm{dL}$ \\
\hline
\end{tabular}




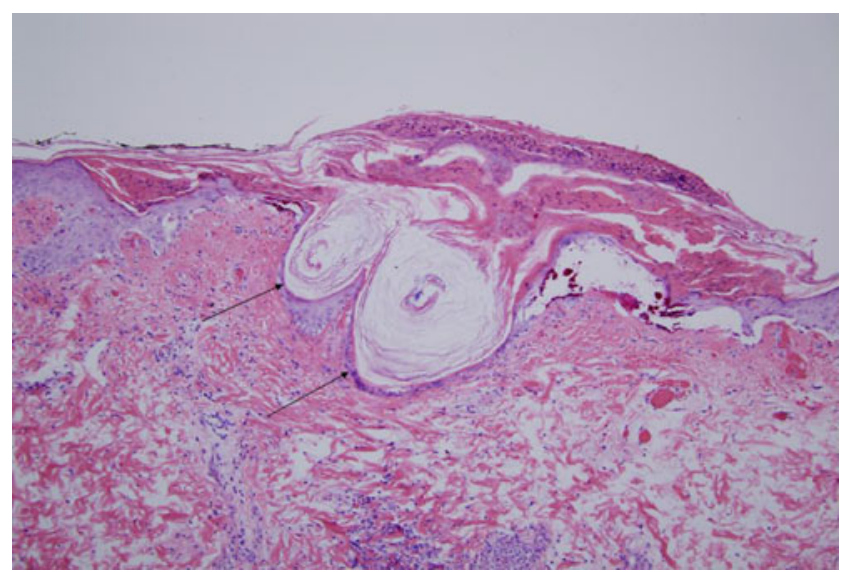

Fig. 1. The biopsy shows a markedly attenuated epidermis surmounted by a compact orthohyperkeratotic scale with attendant follicular plugging (arrows) typical for a discoid lupus-like diathesis.

\section{Clinicopathologic Discussion}

Erythema multiforme in the setting of lupus has been termed RS. RS is a clinical entity first described by Rowell, Beck, and Anderson in 1963 in patients with chronic discoid LE who had annular skin lesions that resembled those of EM, as well as positive anti-La/SSB and anti-Ro/SSA antibodies [9]. Classic EM is an acute, usually self-limited, immune-mediated condition, characteristically presenting with acrally distributed, targetoid skin lesions with three zones of color consisting of a dusky center reflecting necrotic keratinocytes, and an inflammatory zone of skin colored middle rim and erythematous periphery (ESM Picture 1b). It is commonly triggered by viral infections or certain medications, or can rarely present as recurrent or persistent in some cases of infections, inflammatory bowel disease, and malignancy [4]. Typical of RS is a history of recurrent EM lesions without such identifiable triggers in combination with positive autoantibodies (anti-Ro, anti-La, rheumatoid factor, or speckled ANA). In addition, patients described in the original

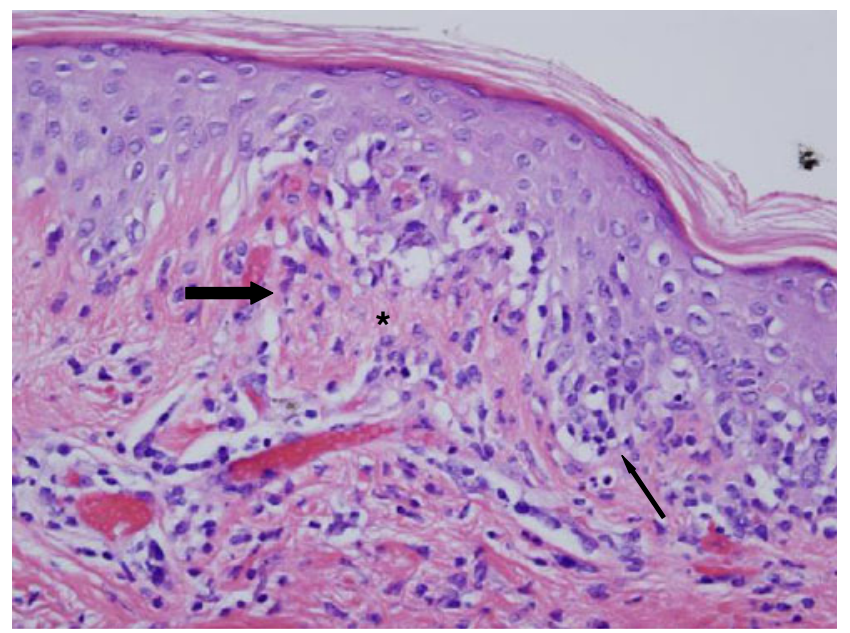

Fig. 2. The biopsy shows an area of cytotoxic interface dermatitis (thin arrow) with prominent lymphocyte satellitosis (thick arrow) around necrotic keratinocytes (asterisk) indicative of a true type IV cytotoxic immune reaction pathogenetically analogous to erythema multiforme. paper were noted to have perniosis (chilblain lupus) lesions, reflective of the acral vasculitis. Rowell et al. presented these clinical and serological findings as distinctive enough to justify a new syndrome (now known as RS); the major and minor criteria were later outlined by Zeitouni et al. [14].

Whether EM-like annular skin lesions seen in SLE patients is an incidental association of a mere coexistence of LE and EM or a separate entity, it is difficult to determine [1]. It has been a matter of debate whether RS is pathogenically distinct from EM in the setting of SLE $[1,5,10,11]$. The majority of cases of RS reported in the literature do not fit the original description of RS, and it may be challenging to clinically differentiate between annular and polycyclic lesions of subacute cutaneous LE and EM [1]. However, specific serologic findings of anti-Ro (SS-A)/La (SS-B) antibodies and rheumatoid factor in association with EM-like lesions are not found in classical EM, nor can any precipitating factors be isolated in RS. The targetoid lesions of EM reflecting the histopathological findings of prominent lymphocyte satellitosis around degenerating keratinocytes in concert with concomitant features of LE, such as mucin deposition and interface dermatitis, are corroborative of a distinctive syndrome.

In RS, patients typically have anti-Ro antibodies, and they often do not report an exogenous disease trigger such as a viral infection or drug therapy. It is possible that the presence of antiRo antibodies may play a pathogenic role in perpetuating a lupus-like diathesis and findings reminiscent of erythema multiforme, evoking a type IV immune response similar to that seen in classic EM triggered by exogenous antigens. In SLE, the main mechanism of the epithelial injury is antibody-dependent cellular cytotoxicity mediated by lymphocytes, rather than a direct antigen epitope response. For example, after UV exposure, epidermal keratinocytes displace nucleosomes to the surface and become antigenic. Anti-ribonuclear antibodies (such as the antiRo antibody seen in this patient) can bind to their surface of keratinocytes, triggering the binding of $\mathrm{T}$ cells bearing $\mathrm{Fc}$ receptors (for example NK cells), and inducing an antibodydependent cellular cytotoxicity that causes the typical lupus features of progressive epidermal destruction and thinning. Concomitantly, the keratinocytes expressing Ro are rendered autoantigenic and invoke a true clonal $\mathrm{T}$ cell response, triggering a significant lymphocytic exocytosis with lymphocyte apposition to necrotic keratinocytes, which is reminiscent of an EM-like pattern of type IV immune response [6, 7, 13]. This may be an important additional pathway of tissue injury that is likely operational in cases of RS whereby an obvious exogenous trigger is not identified.

\section{Hospital Course}

After results of the skin biopsy showing Rowell's syndrome consistent with a lupus-driven inflammatory process, the patient was treated with high-dose corticosteroids $(1 \mathrm{mg} / \mathrm{kg})$, and acyclovir was discontinued. After 8 days of treatment, the rash, oral ulcers, and digital ulcerations almost completely resolved. The patient was maintained on hydroxychloroquine and started on mycophenolate mofetil for steroid-sparing effect; he tolerated a taper of the corticosteroid dose over 6 months, 
down to $4 \mathrm{mg}$ of methylprednisolone orally daily. Over 2 years of follow up, the patient experienced intermittent flares of painful oral ulcers and arthritis, however, without any recurrence of the annular skin lesions (RS).

Although much is known about the autoantibodies that characterize cutaneous and SLE, as well as the abnormal cytokine expression, cellular factors, adhesion molecules, and cell surface receptors involved in the disease, understanding of the exact etiologic factors and pathophysiology of the disorder is lacking. This case report features the clinical syndrome of EM in the setting of SLE, known as RS. It has been a matter of debate in the literature whether RS is a pathogenically distinct entity, and this case report provides a mechanistic theory on how the presence of antiRo antibodies may play a pathogenic role in perpetuating lupus-like diathesis and findings reminiscent of erythema multiforme.

\section{Disclosures}

Conflict of Interest Susan S. Kim, MD, Cynthia Magro, MD, Richard D. Granstein, MD, Anne Bass, MD and Doruk Erkan, MD have declared that they have no conflict of interest.

Human/Animal Rights All procedures followed were in accordance with the ethical standards of the responsible committee on human experimentation (institutional and national) and with the Helsinki Declaration of 1975 , as revised in 2008 (5).

Informed Consent Informed consent was obtained from all patients for being included in the study. Additionally, informed consent was obtained from all patients for which identifying information is included in this article.

Required Author Forms Disclosure forms provided by the authors are available with the online version of this article.

\section{References}

1. Antiga E, Caproni M, Bonciani D, Bonciolini V, Fabbri P. The last word on the so-called "Rowell's syndrome"? Lupus. 2012; 21: 577-585.

2. Brice SL, Leahy MA, Ong L, et al. Examination of non-involved skin, previously involved skin, and peripheral blood for herpes simplex virus DNA in patients with recurrent herpes-associated erythema multiforme. J Cutan Pathol. 1994; 21: 408-412.

3. Crowson AN, Magro CM, Mihm MC. Interface dermatitis. Archives of Pathology \& Laboratory Medicine. 2008; 132: 652-666.

4. Drago F, Parodi A, Rebora A. Persistent erythema multiforme: report of two new cases and review of literature. $J$ Am Acad Dermatol. 1995; 33: 366-369.

5. Fiallo P, Tagliapietra AG, Santoro G, Venturino E. Rowell's syndrome. Int J Derm. 1995; 34: 635-636.

6. Frank MB, McArthur R, Harley JB, Fujisaku A. Anti-Ro(SSA) autoantibodies are associated with $\mathrm{T}$ cell receptor beta genes in systemic lupus erythematosus patients. J Clin Invest. 1990; 85: 33-9.

7. Namekawa T, Kuroda K, Kato T, et al. Identification of Ro(SSA) $52 \mathrm{kDa}$ reactive $\mathrm{T}$ cells in labial salivary glands from patients with Sjögren's syndrome. J Rheumatol. 1995; 22: 2092.

8. Ng PP, Sun YJ, Tan HH, Tan SH. Detection of herpes simplex virus genomic DNA in various subsets of erythema multiforme by polymerase chain reaction. Dermatology. 2003; 207: 349-353.

9. Rowell NR, Beck JS, Anderson JR. Lupus erythematosus and erythema multiforme-like lesions. A syndrome with characteristic immunological abnormalities. Arch Dermatol. 1963; 88: 176-180.

10. Shadid NH, Thissen C, van Marion AMW, Poblete-Gutierrez P, Frank J. Lupus Erythematosus associated with erythema multiforme: Rowell's syndrome. Int J Derm. 2007; 46(suppl 3): 30-32.

11. Shteyngarts AR, Warner MR, Camisa C. Lupus erythematosus associated with erythema multiforme: does Rowell's syndrome exist? J Amer Acad Derm. 1999; 40: 773-777.

12. Sokumbi O, Wetter DA. Clinical features, diagnosis, and treatment of erythema multiforme: a review for the practicing dermatologist. Int J Derm. 2012; 51: 889-902.

13. Sumida T, Namekawa T, Maeda T, Nishioka K. New T-cell epitope of Ro/SS-A $52 \mathrm{kDa}$ protein in labial salivary glands from patients with Sjögren's syndrome. Lancet. 1996; 348: 1667.

14. Zeitouni NC, Funaro D, Cloutier RA, Gagne E, Claveau J. Redefining Rowell's syndrome. Br J Dermatol. 2000; 142: 343-346. 